OPEN ACCESS

Edited by:

Abigail Saffron Tucker

King's College London,

United Kingdom

Reviewed by:

Thomas G. H. Diekwisch,

Texas A\&M University, United States

Javier Catón,

Universidad Complutense de Madrid,

Spain

${ }^{*}$ Correspondence:

Marianna Bei

mbei@partners.org

mbei@mgh.harvard.edu

†These authors have contributed equally to this work

Specialty section: This article was submitted to Craniofacial Biology and Dental

Research,

a section of the journal

Frontiers in Physiology

Received: 12 July 2020 Accepted: 28 September 2020 Published: 26 October 2020

Citation:

Ruspita I, Das P, Xia Y, Kelangi S,

Miyoshi K, Noma T, Snead ML,

D'Souza RN and Bei M (2020) An

Msx2-Sp6-Follistatin Pathway

Operates During Late Stages of Tooth

Development to Control

Amelogenesis.

Front. Physiol. 11:582610.

doi: 10.3389/fphys.2020.582610

\title{
An Msx2-Sp6-Follistatin Pathway Operates During Late Stages of Tooth Development to Control Amelogenesis
}

\author{
Intan Ruspita ${ }^{1,2+}$, Pragnya Das ${ }^{1,3 \dagger}$, Yan Xia ${ }^{1}$, Sarah Kelangi, ${ }^{4,5}$, Keiko Miyoshi6, \\ Takafumi Noma7, Malcolm L. Snead ${ }^{8}$, Rena N. D'Souza ${ }^{9}$ and Marianna Bei ${ }^{4,5 *}$
}

${ }^{1}$ Center for Regenerative and Developmental Biology, The Forsyth Institute, Cambridge, MA, United States, ${ }^{2}$ Department of Prosthodontics, Universitas Gadjah Mada, Yogyakarta, Indonesia, ${ }^{3}$ Division of Neonatology, Cooper University Hospital, Camden, NJ, United States, ${ }^{4}$ Center for Engineering in Medicine, Department of Surgery, Massachusetts General Hospital and Harvard Medical School, Boston, MA, United States, ${ }^{5}$ Shriners Hospital for Children, Boston, MA, United States, ${ }^{6}$ Department of Molecular Biology, Institute of Biomedical Sciences, Tokushima University, Tokushima, Japan, ${ }^{7}$ Faculty of Human Life Studies, Hiroshima Jogakuin University, Hiroshima, Japan, ${ }^{8}$ Center for Craniofacial Molecular Biology, Herman Ostrow School of Dentistry of USC, University of Southern California, Los Angeles, CA, United States, ${ }^{9}$ University of Utah, Salt Lake City, UT, United States

Background: Ameloblasts are epithelially derived cells responsible for enamel formation through a process known as amelogenesis. Amongst the several transcription factors that are expressed during amelogenesis, both Msx2 and Sp6 transcription factors play important role. Msx2 and Sp6 mouse mutants, exhibit similar amelogenesis defects, namely enamel hypoplasia, while humans with amelogenesis imperfecta (Al) carry mutations in the human homologues of MSX2 or SP6 genes. These across species similarities in function indicate that these two transcription factors may reside in the same developmental pathway. In this paper, we test whether they work in a coordinated manner to exert their effect during amelogenesis.

Methods: Two different dental epithelial cell lines, the mouse LS8 and the rat G5 were used for either overexpression or silencing of Msx2 or Sp6 or both. Msx2 mutant mouse embryos or pups were used for in vivo studies. In situ hybridization, semiquantitative and quantitative real time PCR were employed to study gene expression pattern. MatInspector was used to identify several potential putative Msx2 binding sites upstream of the murine Sp6 promoter region. Chromatin Immunoprecipitation (chIP) was used to confirm the binding of Msx2 to Sp6 promoter at the putative sites.

Results: Using the above methods we identified that (i) Msx2 and Sp6 exhibit overlapping expression in secretory ameloblasts, (ii) Sp6 expression is reduced in the Msx2 mouse mutant secretoty ameloblasts, and (iii) that Msx2, like Sp6 inhibits follistatin expression. Specifically, our loss-of function studies by silencing Msx2 and/or Sp6 in mouse dental epithelial (LS8) cells showed significant downregulation of Sp6 but upregulation of Fst expression. Transient transfection of Msx2 overexpression plasmid, 
up-regulated Sp6 and downregulated Fst expression. Additionally, using MatInspector, we identified several potential putative Msx2 binding sites, $3.5 \mathrm{~kb}$ upstream of the murine Sp6 promoter region. By chIP, we confirmed the binding of Msx2 to Sp6 promoter at these sites, thus suggesting that Sp6 is a direct target of Msx2.

Conclusion: Collectively, these results show that Sp6 and Msx2 work in a concerted manner to form part of a network of transcription factors that operate during later stages of tooth development controlling ameloblast life cycle and amelogenesis.

Keywords: Msx2, Sp6, follistatin, dental epithelial cells, in situ hybridization, chromatin immunoprecipitation

\section{INTRODUCTION}

The development of teeth depends on cell interactions between epithelium and mesenchyme that leads to the differentiation of cells derived from mesenchyme into odontoblasts and of cells derived from epithelium into ameloblasts (Kollar and Lumsden, 1979; Thesleff and Nieminen, 2005). The process of epithelial cells differentiating into functional ameloblasts is timedependent and through this process several morphologic changes occur known as: (i) the inductive stage (pre-ameloblasts); (ii) the initial-secretory stage; (iii) the secretory stage; and (iv) the maturation stage (Nanci, 2007). Pre-secretory, secretory, and mature ameloblasts express several proteins, including secreted proteins, enzymes, signaling molecules, cell-cell adhesion molecules, and transcription factors (reviewed in Wright et al., 2000, 2015; Aldred et al., 2003; Wright, 2006; Hu et al., 2008; Nakamura et al., 2008; Bei, 2009a,b; Bartlett, 2013; Habelitz, 2015; Shin et al., 2020).

Studies in animal models and humans have shown that Msx2 and Sp6 transcription factors play important role during amelogenesis. Specifically, in mice lacking the homeobox gene Msx2 "the ameloblasts reach the secretory stage of their differentiation process, but only sparse amounts of enamel matrix are deposited" (Satokata et al., 2000; Bei et al., 2004; Babajko et al., 2014). In a case of syndromic amelogenesis imperfecta (AI) sequence analysis of the human homolog of MSX2 gene identified a missense mutation of $\mathrm{T} 447 \mathrm{C}$, further indicating the important role of $M s x 2$ during amelogenesis (Suda et al., 2006). Expression studies have also indicated that Msx2 is required for the expression of important secreted proteins and cell-cell adhesion molecules for amelogenesis, such as laminin 5 alpha 3 , amelogenin, and enamelin (Bei et al., 2004; Ruspita et al., 2008; Molla et al., 2010).

Specificity protein $6(S p 6)$ is another transcription factor that, like $M s \times 2$, is expressed by secretory ameloblasts and when its function is eliminated in mice amelogenesis is affected. The Sp6 mutant mice among other phenotypes exhibit enamel hypoplasia (Nakamura et al., 2008; Utami et al., 2011). Recently, in a Caucasian family with autosomal dominant hypoplastic AI, a missense protein change, p.(Ala273Lys), is identified in SP6, the gene encoding the SP6 transcription factor (Smith et al., 2020). The authors have also "identified a potential SP6 binding motif in the $A M B N$ proximal promoter sequence and showed that wildtype (WT) SP6 binds more strongly to it than the mutant protein," further indicating the important role of Sp6 in amelogenesis
(Smith et al., 2020). Earlier studies indicate that Sp6 promotes amelogenesis in vitro through inhibition of follistatin (Fst) gene which is a soluble extracellular inhibitor of TGF $\beta$ superfamily and is involved in differentiation of secretory ameloblasts during tooth development (Ruspita et al., 2008). In addition, "overexpression of follistatin in the dental epithelium inhibits ameloblast differentiation in transgenic mouse incisors, whereas in follistatin knockout mice, ameloblasts differentiate ectopically on the lingual enamel-free surface" (Wang et al., 2004).

Based on the above, the role of Msx2 and Sp6 genes in amelogenesis is important. Here, we study the interplay between $M s x 2$, Sp6, and Fst and we show that (i) $S p 6$ expression is reduced in the $M s \times 2$ mouse mutant secretoty ameloblasts, (ii) $M s x 2$, like Sp6 inhibits follistatin expression in vitro, (iii) Sp6 and follistatin are early response genes whose expression is under the control of Msx2, and that (iv) Msx2 binds to Sp6 promoter in vitro, suggesting that $S p 6$ is a direct target of $M s \times 2$. These results raise the possibility that these transcription factors interact closely with each other and within a common molecular cascade.

\section{MATERIALS AND METHODS}

\section{Cell Culture}

Two different dental epithelial cell lines were used in the present study - the rat dental epithelial cell line (G5), generously provided by Dr. Takafumi Noma, Dental School of Tokushima University, Japan and the mouse dental epithelial cell line (LS8) kindly provided by Dr. Malcolm Snead, USC, CA, United States. Both cell lines were maintained in high-glucose Dulbecco's modified Eagle's medium (Gibco, Grand Island, NY, United States), containing $100 \mathrm{U} / \mathrm{ml}$ penicillin, $100 \mathrm{mg} / \mathrm{ml}$ streptomycin, and $10 \%$ fetal bovine serum (Gibco, Grand Island, NY, United States) at $37^{\circ} \mathrm{C}$ in $5 \% \mathrm{CO}_{2}$ humidified atmosphere following the standard protocols (Ruspita et al., 2008; Chang et al., 2017)

\section{Gain-of-Function and Loss-of-Function Studies}

For overexpression of Msx2, LS8, and G5 cells were transfected with pCMVtag2-Flag-Msx2, and then cultured for 48-72 h following which total RNA was isolated from the cells using Trizol (Qiagen, MD, United States). An empty vector (pCMVtag2) served as a negative control for gain-of-function studies. 
For loss of function of $M s \times 2$, commercially available small interfering RNA for $M s x 2$ (Msx2-siRNA) was purchased from Santa Cruz Biotechnology (Santa Cruz, CA, United States). We used the following oligonucliotides, sense sequence 5'CAGCUCUCUGAACCUUAC 3' (sc-43947). As negative control we used a scramble sequence that will not lead to the specific degradation of any known cellular mRNA: sense scramble control 5'UUCUCCGAACGUGUCACG 3' (sc-37007). To prepare lipid-siRNA complexes, the siRNA (80 pmol) in $100 \mu \mathrm{l}$ of transfection medium (sc-36868) and $5 \mu \mathrm{l}$ of siRNA transfection reagent (sc-29528) in $100 \mu \mathrm{l}$ of transfection medium were combined, incubated for $30 \mathrm{~min}$ at $25^{\circ} \mathrm{C}$, and then diluted with $800 \mu \mathrm{l}$ of transfection medium. Cells were rinsed once with serum-free DMEM/F12, and $1000 \mu \mathrm{l}$ of lipid-siRNA mixturedescribed above-was applied per well. After incubation for $6 \mathrm{~h}$ at $37^{\circ} \mathrm{C}$ in a humidified $5 \% \mathrm{CO}_{2}$ cell chamber, an additional $1 \mathrm{ml}$ of 20\% FBS in DMEM/F12 was added per well, and lipofection was allowed to continue overnight. The following day, lipofection media was aspirated, and transfected monolayer cells refed with fresh 10\% FBS in DMEM/F12. After 48 and $72 \mathrm{~h}$, total cellular RNA was harvested for reverse-transcriptase-polymerase chain reaction (RT-PCR) analysis. For lentiviral (ShRNA) gene knock down assay, we obtained plasmids containing the sequences for Msx2-shRNA, Sp6-shRNA and non-target scramble shRNA from Sigma Aldrich (St. Louis, MO, United States). LS8 cells were seeded into six-well culture plates and cultured in DMEM/F12 containing $10 \%$ fetal bovine serum without antibiotic. Upon $80 \%$ confluency, cells were infected with lentiviruses with a MOI (multiplicity of infection) $=5$ and selected for stable integration with $1 \mu \mathrm{g} / \mathrm{ml}$ puromycin.

\section{Time Dependent Assay}

LS8 cells were transfected with pCMVtag2-Flag-Msx2 (Invitrogen, United States) and then cultured for up to $48 \mathrm{~h}$. The cells were harvested at 4 and $16 \mathrm{~h}$ for RNA isolation and subjected to real time qPCR analysis to check for expression of Sp6 and Fst.

\section{RNA Extraction and Reverse-Transcriptase-Polymerase Chain Reaction}

Total RNA was isolated from cultured cells after the desired time points using the standard procedure by TRIZOL (Qiagen) method. First-strand cDNA was generated from $1 \mu \mathrm{g}$ of total RNA using quantitect, RT kit (Qiagen, Valencia, CA, United States) in total of $20 \mu \mathrm{l}$ according to the manufacturer's instructions. Semi-quantitative PCR was performed on $1 \mu \mathrm{l}$ of RT product in $20 \mu \mathrm{l}$ of reaction mixture to check for expression of Msx2, Sp6, and Fst. Gapdh was used as the loading control. The PCR products were analyzed on $1.5 \%$ agarose gel. The primer sequences are listed in Supplementary Table 1.

\section{Real-Time Quantitative PCR}

For real-time quantitative PCR, total RNA from was isolated from cultured cells after the desired time points using the standard procedure by TRIZOL (Qiagen) method and reverse transcriptions were performed using qScript cDNA synthesis kit (Quanta Biosciences, Gaithersburg, MD, United States). Quantitative PCR was carried out in LightCycler and LightCycler-Faststart DNA Master SYBR Green I (Roche Diagnostics, Switzerland). The expression level of each sample was normalized to glyceraldehyde-3phosphate dehydrogenase (GAPDH) mRNA expression. The primer sequences are listed in Supplementary Table 1.

\section{Mice and Genotyping}

All animal studies and experimental procedures were conducted in accordance to the guidelines for the care and use of laboratory animals by the Forsyth Institute, Cambridge, MA and Massahusetts General Hospital, Boston, MA. Embryos and postnatal pups (E18.5, P1 and P3) were collected from matings of Msx2 heterozygous animals maintained in BALB/c background. The day of plug discovery was designated as embryonic day 0.5 (E0.5). Genotyping was performed as previously described (Bei et al., 2004). Age matched wildtype pups and/or embryos served as the appropriate controls.

\section{In situ Hybridization}

Embryonic Day 18.5 embryos and postnatal animals (P1, P3) were collected and heads decapitated for making coronal and sagittal sections. E18.5, P1 and P3 samples were immediately fixed in $4 \%$ paraformadehyde. All samples were then dehydrated through graded ethanol series, embedded in paraffin, sectioned at $8 \mu \mathrm{m}$ and processed for in situ hybridization (ISH), as previously described (Bei and Maas, 1998). Murine Sp6 and Fst antisense probes were purchased from IDT (IA, United States) and labeled with DIG-UTP (Roche) following the manufacturer's instructions. The sense probes for both genes were used as a negative control. In situ hybridization was performed as previously described (Bei et al., 2004)

\section{Chromatin Immunoprecipitation Assay}

Chromatin immunoprecipitation (chIP) was performed using the EZ-Magna chip kit (Millipore, Billerica, MA, United States) according to the manufacturer's instructions. Forty-eight hours after transfection with pCMV-FLAG-Msx2 expression plasmid, LS8 cells were fixed and crosslinked with $1 \%$ (v/v) formaldehyde at $37^{\circ} \mathrm{C}$ for $10 \mathrm{~min}$. Crosslinking was stopped by adding glycine to a final concentration of $125 \mathrm{mM}$, followed by washing with cold PBS. After sonication chromatin was incubated with magnetic beads conjugated to either $1 \mu \mathrm{g}$ of monoclonal anti-Flag antibody (Sigma) or $1 \mu \mathrm{g}$ of normal rabbit IgG (Sigma) antibody. Immunoprecipitated chromatin was reverse crosslinked and washed before DNA extraction. Polymerase A was used as a positive control while IgG was used as the negative control. Finally, the immunoprecipitated DNA and the corresponding non-immunoprecipitated DNA (input) was subjected to PCR using different set of forward and reverse primers, specific for the different putative binding regions, and analyzed on $1.5 \%$ agarose gel. The primers used for the different putative binding sites are listed in Supplementary Table 1. 


\section{In silico Analysis of Promoter Binding Sites}

UCSC MatInspector software was used to predict the putative promoter binding regions for Msx2. Primers were designed from these predicted regions for chIP followed by PCR amplification using these primers, using Primer 3 database.

\section{Imaging and Densitometric Quantification}

The imaging for ISH was done using Olympus microscope while densitometric quantification of semi-quantitative RT-PCR bands were done using Image ( $\mathrm{NIH}$, version 5).

\section{Statistics}

Each cell culture experiment was replicated three times. For ISH, a minimum of 3-4 mice pups were used. Statistics was done using one-way ANOVA or students $t$-tailed test, wherever applicable using GraphPad prism (version 7, CA). $P$ value of $<0.05$ was considered statistically significant.

\section{RESULTS}

\section{Msx2 Differentially Regulates the Expression of Genes Involved in Amelogenesis}

There are several genes known to be involved in amelogenesis (Bei, 2009b; Wright et al., 2015). To test whether Msx2 regulates the expression of some of these genes, we performed semiquantitative RT-PCR after overexpressing Msx2 in LS8 (Figure 1) and G5 cells (data not shown). The ameloblast-like cell LS8 cell line, derived from murine EO epithelium, and the G5 cell line, derived from rat dental epithelial derived ameloblast-lineage clone, both, constitute ideal cell systems to test gene regulation for the following reasons. (Chen et al., 1992; Xu et al., 2006; Abe et al., 2007; Ruspita et al., 2008). The LS8 and G5 "cells express many of the genes specific for amelogenesis, such as ameloblastin, amelogenin, and enamelin, at sufficiently high levels, they have been used for many in vitro studies of amelogenesis, including gene promoter analysis and LS8 cells, in particular, produce an enamel extracellular matrix that is similar to authentic enamel after treatment with peptide amphiphiles" (Zhou et al., 2000; Huang et al., 2008). After overexpressing Msx2 in both cells lines, we show that the expression of Sp6, Sp3, Sprouty 2, Connexin 43, Wnt3, Tgfb1 and Enam (enamelin), Laminin 5 alpha 3 (lama3), as well as Msx2 itself, is up-regulated in the Msx2 overexpressing cells. In contrast, the expression of Tbx1, Amel (amelogenin), Fst (Follistatin) is downregulated in the Msx2 overexpressing cells, while Ambn (ameloblastin) expression is partially diminished, almost not affected (Figure 1). These results further confirm previous results where we and others showed that Msx2 is required for the regulation of Lama3, Amel, Enam and Ambn gene expression (Zhou et al., 2000; Bei et al., 2004; Ruspita et al., 2008; Molla et al., 2010; Bei, unpublished). We also show for the first time that $M s \times 2$ is required for the regulation of $S p 6, S p 3$, Sprouty 2, Connexin 43, Wnt3, Tgfb1, Tbx1, and Fst (Follistatin)

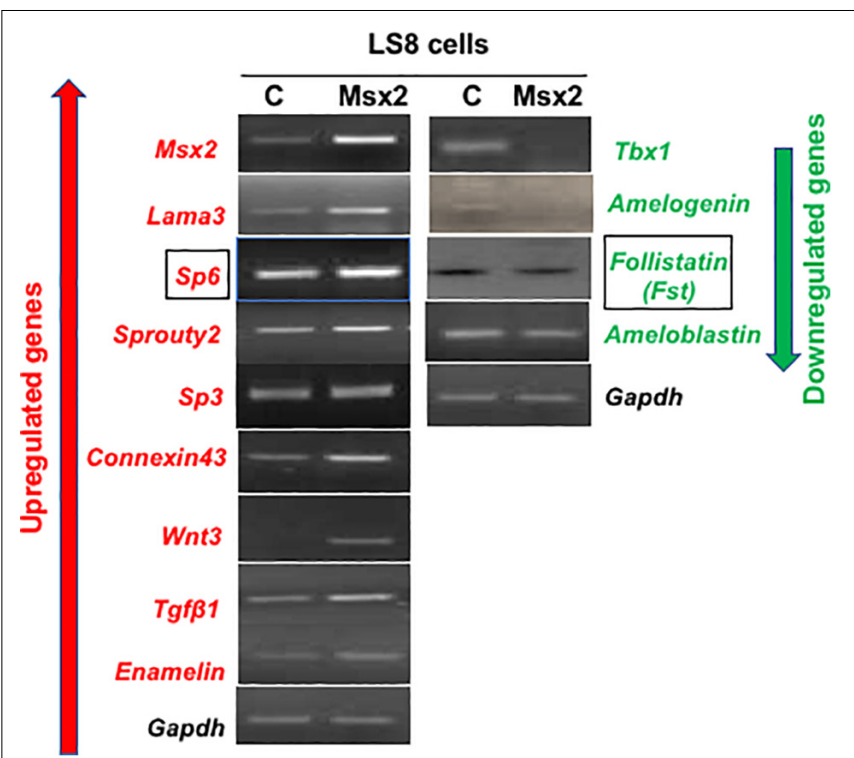

FIGURE 1 | Screening of representative genes involved in amelogenesis: LS8 cells were transfected with pCMVtag2-Flag-Msx2 then cultured for $48 \mathrm{~h}$. Total RNA was isolated from the cells and subjected to reverse transcription PCR analysis. Upregulated genes are shown in red while downregulated ones are shown in green. The expression of Sp6, Sp3, Sprouty 2, Connexin 43, Wnt3, Tgfb 1 and Enam (enamelin), Laminin 5 alpha 3 (lama3), as well as Msx2 itself, is up-regulated in the Msx2 overexpressing cells. In contrast, the expression of Tbx1, Amel (amelogenin), Fst (Follistatin) is downregulated in the Msx2 overexpressing cells, while Ambn (ameloblastin) expression is partially diminished, almost not affected. Gapdh is the housekeeping gene. C, cells transfected with control vector only; Msx2, cells are transfected with pCMVtag2-Flag-Msx2.

gene expression. For the purposes of this study, we will focus on Sp6 and Fst regulation, only.

\section{The Expression of Sp6 and Fst Is Modulated Early in Response to Msx2}

The previous results indicate that $S p 6$ expression is upregulated while Fst expression is downregulated, upon Msx2 overexpression. To determine the kinetics of Sp6 and Fst (Follistatin) gene expression, we performed a time dependent assay to ascertain whether their expression is modulated early in response to Msx2 upregulation (Figure 2). LS8 cells were transfected with $M s x 2$ over-expression plasmid for two time points, 4 and 16 hours. Total RNA was isolated from the cells and subjected to qPCR analysis. Sp6 and Fst could be detected as early as $4 \mathrm{~h}$ after transfection by real time qPCR (Figure 2). By $16 \mathrm{~h}$, there was a significant increase in the expression of Sp6 (Figure 2A) with a corresponding decrease of Fst expression (Figure 2B). We have not seen any significant response earlier than $4 \mathrm{~h}$, thus these results indicate that both Sp6 and follistatin are secondary early response genes to Msx2 (response after $4 \mathrm{~h}$ of $M s x 2$ overexpression). These experiments have not been performed in the presence of the protein synthesis inhibitor, cycloheximide and thus, we do not know whether the secondary 


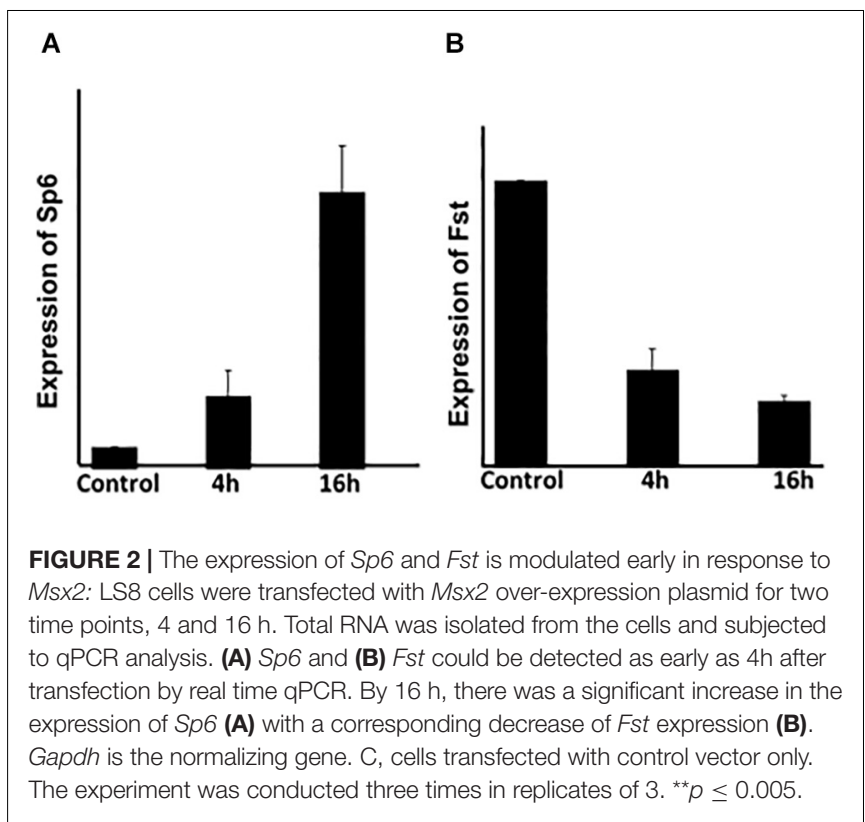

response of Sp6 and Fst genes require de novo protein synthesis for transcription.

\section{Loss of Function of Msx2 and Sp6 in LS8 Ameloblast-Derived Cells}

We have shown that overexpression of Msx2 in both LS8 (Figures 2, 3A) and G5 cells (Figure 3A) leads to a significant increase of $M s \times 2$ in both cell lines-indicating that the transfection efficiency is quite successful-with concomitant increase of Sp6 and decrease of Fst expressions (Figure 3A). To test whether the opposite holds true, we tested the effects of acute knockdown of Msx2 in LS8 ameloblast-derived cells, and compared to what happens in development where Msx2 is permanently absent in the Msx2-null mice (Figure 4). For the knock down experiment, we used siRNA technology in LS8 cells (Figure 3B). After 48 and $72 \mathrm{~h}$ transfection the cells were subjected to RT-PCR. We found that upon silencing of Msx2, Sp6 was downregulated while Fst expression was upregulated, further suggesting that Sp6 requires Msx2 for its expression and that Msx2 inhibits Fst expression (Figure 3B). In addition, we used lentiviral shRNA mediated approach to assess the direct effects of silencing Msx2 and Sp6 genes. Specifically, the LS8 cells were infected with mouse Msx2shRNA, or Sp6shRNA or both lentiviral transduction particles. qPCR shows that (i) Msx2shRNA or Sp6shRNA lentiviral transduction particles alone effectively reduce each other's expression in LS8 cells, while they increase expression of Fst compared to control shRNA treated cells; (ii) that both Msx2shRNA plus Sp6shRNA lentiviral transduction particles abolished Sp6 and Msx2 expression in LS8 cells and increased Fst expression comparing with control shRNA treated cells (Figure 3C).

The loss of function along with the gain of function results show for the first time that Msx2 is required for Sp6 expression and that Sp6 is required for Msx2 expression. This result suggests that these two transcription factors may interact at the molecular level to control each other's transcriptional output. We also show that Msx2 inhibits Fst expression and confirmed that Sp6 inhibits Fst expression (Ruspita et al., 2008). In addition, we show that Msx2 and Sp6 act synergistically to control the level of Fst inhibition, since when we silenced both Msx2 and Sp6, at the same time, the inhibition of Fst was less robust compared to silencing by $M s \times 2$ or Sp6 alone. The latter result is of particular interest as it suggests that the synergistic interaction of Msx2 with Sp6 may alleviate each other's inhibitory effect on Fst by allowing one, or more than one, transcription activators to exert their function and thus regulating Fst's gene dosage.

\section{Msx2 Is Essential for Sp6 Gene Expression During Late Tooth Development}

To determine whether $M s \times 2$ is required for Sp6 regulation and whether this requirement is associated with the defect in amelogenesis, in situ hybridization was performed in wild type and Msx2 deficient mouse molar tooth germs at E.18.5, postnatal day 1 (P1) and postnatal day 3 (P3) (Figure 4). Msx2 is expressed at very low levels by pre-ameloblasts (E18.5-P1) but is highly expressed in secretory ameloblasts (P3) (MacKenzie et al., 1992; Gritli-Linde et al., 2002; Bei et al., 2004; Bei, 2009a,b). Partial diminution of Sp6 expression is observed in Msx2 deficient tooth germs at P1 compared to wild type (Figures $4 \mathrm{~A}, \mathbf{B}$ ). In contrast, a dramatic reduction of Sp6 expression, is observed in Msx2 deficient ameloblasts compared to wild type at P3, when ameloblasts are at their secretory stage (Figures 4C,D). This result indicates that $S p 6$ requires $M s \times 2$ for its expression in the secretory stage ameloblasts, and is consistent with the in vitro loss and gain of function studies.

Follistatin (Fst) starts to be co-expressed with Msx2 at the early bell stage of tooth development. Although it seems to be expressed widely in the dental epithelial organ, its expression is more concentrated in the inner enamel epithelium (iee). As development proceeds however, and the inner enamel epithelium cells become pre-secretory ameloblasts and, later on, secretory ameloblasts, Fst ceases to be expressed (Wang et al., 2004; Bei, 2009b). In the absence of Msx2, follistatin is expressed throughout the dental epithelium at the bell stage and its expression is increased in the inner dental epithelium (data not shown). This result is consistent with the in vitro loss of function and gain of function studies.

\section{Msx2 Directly Binds to Msx2 Recognition Sites on the Sp6 Promoter}

The loss and/or gain-of-function studies along with the in vivo experiments using the Msx2 mouse mutants revealed that Msx2 is required for the expression of $S p 6$ in the secretory ameloblasts, during amelogenesis. Computational sequence analysis of the nucleotides in the proximal $3.5 \mathrm{~kb}$ of the murine Sp6 promoter region revealed the presence of 6 fully conserved Msx 2 binding sites upstream from the transcription initiation site in the mouse (Supplementary Figure 1). To determine whether Msx2 binds to any of these sites and, therefore, directly 
A
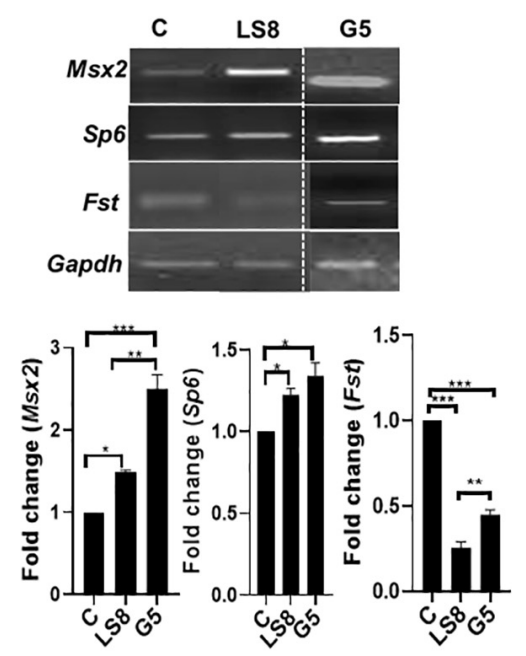

B
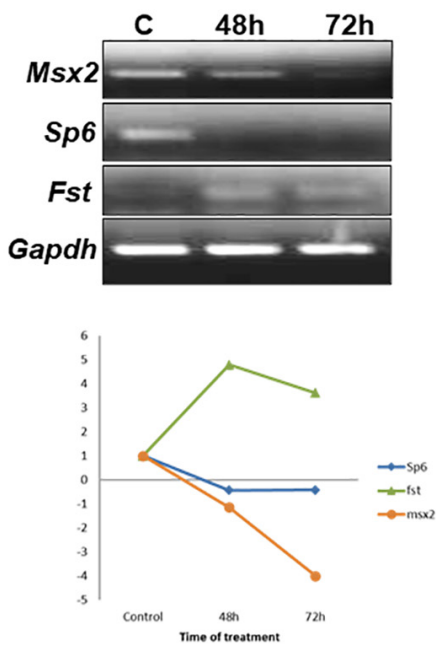
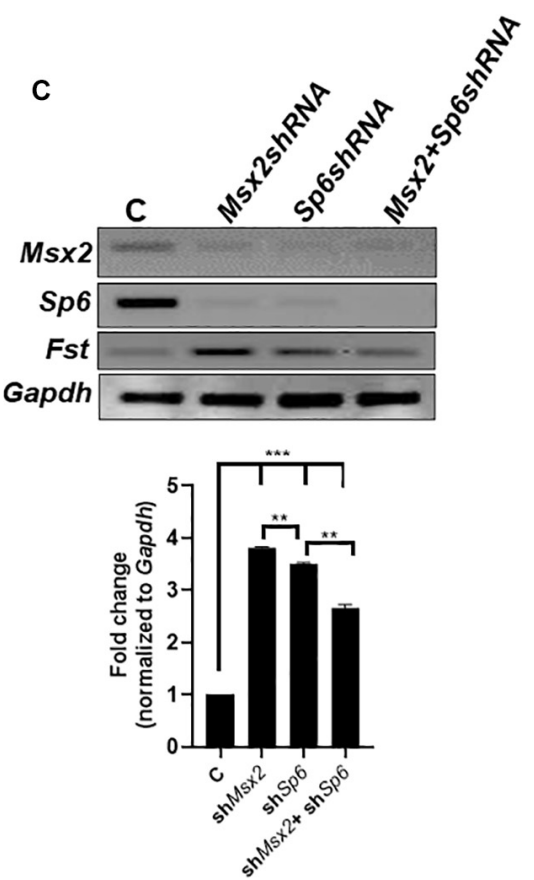

FIGURE 3 | Loss of function of Msx2 and Sp6 in LS8 ameloblast-derived cells: (A) Both LS8 and G5 cells were overexpressed with Msx2 over-expression plasmid. Representative RT-PCR showing Sp6 is upregulated whereas Fst is downregulated in both cell lines after Msx2 overexpression. Bottom panel shows densitometric quantification of the bands. (B) The knockdown of Msx2 with siRNA relative to scrambled control shows downregulation of Msx2 and Sp6 and upregulation of Fst, in 48 and $72 \mathrm{~h}$. RT-PCR results were normalized to Gapdh that served as an internal control and expression levels were relative to scrambled controls. ${ }^{\star \star} P<0.01$. Bottom panel shows a graph describing the trend of genes' expression. (C) Lentiviral (ShRNA) gene knockdown assay, where Msx2-shRNA and Sp6-shRNA, and non-target-shRNA infected LS8 cells further confirm the siRNA results. QPCR results were normalized to Gapdh. Bottom panel shows densitometric quantification of the bands. Experiments were done in triplicates. ${ }^{* *} p \leq 0.005 ;{ }^{* \star *} p \leq 0.0001$.

regulates $S p 6$, chromatin immuno-precipitation was performed with exogenously expressed Msx2-FLAG. Immunoprecipitated chromatin fragments (IP samples) and non-immunoprecipitated samples (1\% input) were subjected to PCR analysis using specific primers spanning the six binding sites. PCR amplifications showed that Msx2 binds directly to four out of six sites carrying the conserved motif (TAAT) in the endogenous promoter of the mouse Sp6 gene (Supplementary Figure S1). This result demonstrates that Msx2 binds directly to the proximal Sp6 promoter in vitro.

In sum, we show that (i) Msx2 and Sp6 exhibit overlapping expression in secretory ameloblasts; (ii) they regulate each other's expression; (iii) Msx2, like Sp6 alone or in coordination with Sp6 inhibits follistatin expression; and (iv) Msx2 binds directly to Sp6 promoter, suggesting that $S p 6$ is a direct target of Msx2.

Collectively, these results raise the possibility that the Sp6 and Msx2 transcription factors interact closely with each other and work in a concerted manner within a common molecular cascade to form part of a network of transcription factors that control ameloblast life cycle and amelogenesis.

\section{DISCUSSION}

Of the several transcription factors, Msx2 and Sp6 constitute key players of amelogenesis. Both, Msx2 and Sp6 mouse mutants, exhibit enamel hypoplasia, while humans with AI carry mutations in the human homologues of MSX2 or SP6 genes (Satokata et al., 2000; Bei et al., 2004; Suda et al., 2006; Nakamura et al., 2008; Utami et al., 2011; Babajko et al., 2014; Smith et al., 2020). These similarities in function indicate that these two transcription factors may reside in the same developmental pathway.

In this paper, we show that Msx2 and Sp6 transcription factors reside in the same developmental pathway and that they work in a coordinated manner to regulate the expression of follistatin $(F s t)$, a signaling molecule that also controls enamel formation (Wang et al., 2004).

\section{Msx2 and Sp6 Transcription Factors Require Each Other to Exert Their Function}

Our gain of function, loss of function, time dependent assay and in vivo data demonstrate that Sp6 requires Msx2 for its expression (Figure 5). Our loss of function experiments indicate that $M s x 2$ also requires Sp6 for its expression, indicating that these two genes reside in the same genetic pathway and that these two transcription factors may interact at the molecular level to control each other's transcriptional output (Figure 5). Characterization of Sp6 promoter for Msx 2 binding sites revealed six putative Msx2 binding sites and our ChIP experiments 


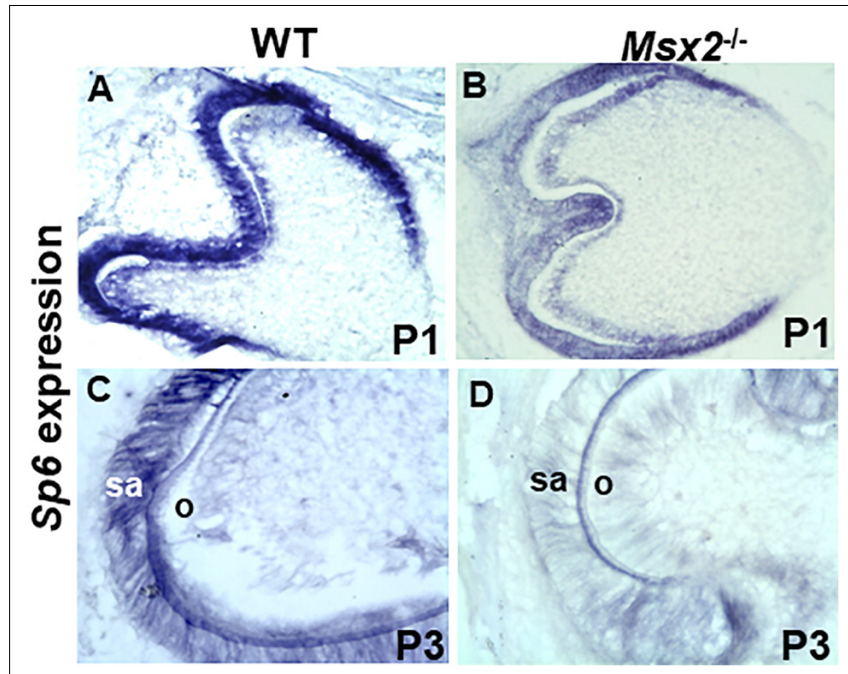

FIGURE 4 | Msx2 is essential for Sp6 gene expression during late stage tooth development: In situ hybridization analyses of transcripts in wild type $(\mathbf{A}, \mathbf{C})$ and Msx2 deficient $(\mathbf{B}, \mathbf{D})$ first lower molar teeth at postnatal day P1 $\mathbf{( A , B )}$ and postnatal day P3 $(\mathbf{C}, \mathbf{D})$. Expression of Sp6 is reduced (B) relative to wild type (A). In contrast, a dramatic reduction of Sp6 expression, is observed in Msx2 deficient ameloblasts (D) compared to wild type at P3 (C). sa, secretory ameloblasts. Scale: X400 $(N=4)$.

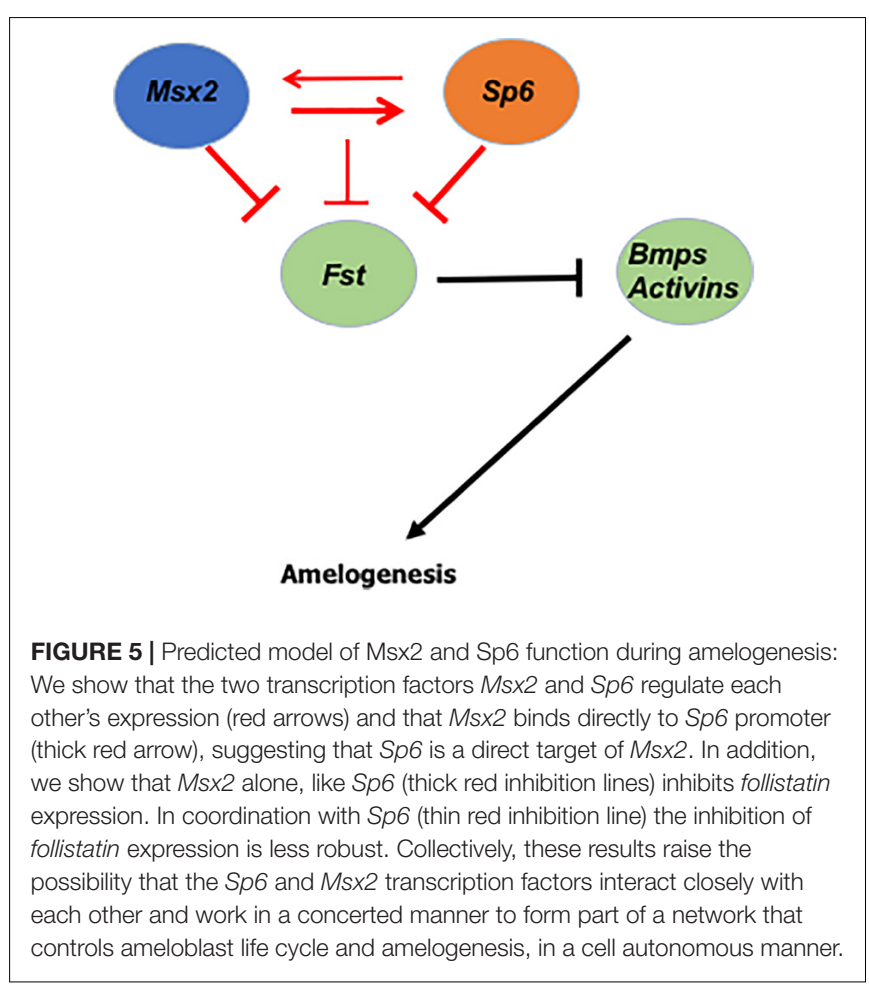

provided evidence that Msx2 binds directly to $S p 6$ promoter to four out of six sites, suggesting that $S p 6$ is a direct target for Msx2 and that Msx2 may promote Sp6 expression directly acting as an activator of $S p 6$ expression (Figure 5). Msx1 and Msx2 transcription factors are known to act as repressors
(Catron et al., 1993, 1995; Zhang et al., 1997), but, consistent to our results recent findings in other developmental systems demonstrate that Msx1 and Msx2 may act as transcriptional activators, as well (Duval et al., 2014).

The fact that Msx2 binds directly to the Sp6 promoter does not rule out the possibility that these two transcription factors may interact with each other physically and/or in vivo via a proteinprotein interaction mechanism. Consistent with this idea, we have shown that Msx2 interacts in vitro and in vivo with another member of the $\mathrm{Sp}$ family of transcription factors, the Sp3 (Zhao et al., 2013). Interstingly, $S p 3$ homozygous null mice, like $S p 6$ null mice, exhibit a hypoplastic phenotype in both, dentine and enamel matrices (Bouwman et al., 2000).

Another interesting finding is that Msx2 seems to regulate its own expression. The gain of function experiments indicate that Msx2 is required for its own expression. This is consistent with other studies showing that Msx2 auto-regulates its promoter and simultaneously represses Dlx2 transcription factor activation in LS8 ameloblast-like cells (Diamond et al., 2006). Here, we show that Msx2 is required for its own expression potentially via an autoregulation mechanism to activate the Sp6 transcription factor. As transcription factors are known to operate sometimes through a feed-forward positive autoregulation mechanism, we can hypothesize that in this case Msx2 activates expression of Sp6, followed potentially by auto-regulatory binding to maintain expression of both genes at a certain level for specific time. To our knowledge, it is not known whether Sp6 operates through an auto-regulatory mechanism, like Msx2 does. Considering, however, that transfection of Sp6 promoted dental epithelial cell differentiation into ameloblasts, by controlling the rate of proliferation of inner enamel epithelium (Nakamura et al., 2008; Ruspita et al., 2008), it would be interesting to see in the future, whether these two transcription factors sustain each other's expression through positive autoregulation. Positive autoregulation is a process where a transcription factor either directly or indirectly activates its own expression, resulting in continuation of transcription in the absence of the factors that started its expression.

\section{Follistatin Expression Is Inhibited by, Both, Msx2 and Sp6 to Promote Amelogenesis}

In vivo studies have clearly demonstrated that "Follistatin is essential for enamel-free area formation by preventing ameloblast differentiation" (Wang et al., 2004). "Overexpression of follistatin, a BMP inhibitor, in the epithelium abrogates ameloblast differentiation. The K14-follistatin mice lack enamel, the ameloblasts fail to form and they do not express enamel specific markers" (Wang et al., 2004). In contrast, in the follistatin knockout mice, functional ameloblasts differentiated on the normally enamel-free surface (Wang et al., 2004). In addition, "experiments on cultured tooth explants suggest that the mechanism by which follistatin prevents ameloblast differentiation is by inhibiting the ameloblast-inducing activity of BMP4 from the underlying odontoblasts. Thus, follistatin 
controls ameloblast differentiation in a cell-autonomous manner by integrating the effect of a non-cell-autonomous signal, that of BMP4 from odontoblasts" (Wang et al., 2004).

In the secretory ameloblasts, it is known that Sp6 inhibits Fst expression (Ruspita et al., 2008). Here, we show for the first time that, like Sp6, Msx2 inhibits Fst expression in the secretory ameloblasts and confirmed the previous result by Ruspita et al. (2008). In addition, we show that Msx 2 and Sp6 act synergistically to control the level of Fst inhibition, since when we silenced both Msx2 and Sp6, at the same time, the inhibition of Fst was less robust compared to silencing by $M s \times 2$ or Sp6 alone (Figure 5). The latter result is of particular interest as it suggests that Msx2's synergistic interaction with Sp6 may alleviate each other's inhibitory effect on Fst by allowing a third or other transcription activators to exert their function and thus regulating follistatin's final gene dosage (Figure 5).

\section{A Cell Autonomous Pathway of Msx2, Sp6, and Fst Operate to Ensure Enamel Formation}

As mentioned above, the role of follistatin in preventing enamel to be formed by inhibiting the ameloblast-inducing activity of BMP4 from the underlying odontoblasts is well known (Wang et al., 2004). What this paper shows, is that Msx2 and Sp6 transcription factors coordinately function by regulating each other's expression to ensure that the expression of follistatin is inhibited and that ameloblasts secrete enamel (Figure 5). Follistatin is expressed in the inner enamel epithelium to ensure proliferation of the cells and it does so by inhibiting the ameloblast-inducing activity of BMP4 from the underlying odontoblasts (Wang et al., 2004; Bei, 2009b). For enamel to be formed, follistatin needs to cease to be expressed, so that BMP4 from underlying odontoblasts is able to induce enamel formation. Thus, controlling the timing of follistatin's switch, from on to off, is extremely important. In light of our recent findings, we propose that Msx2 and Sp6's coordinated action controls either the cease of follistatin's expression or the reduction of its level in order to promote enamel deposition, in a cell autonomous manner (Figure 5).

\section{DATA AVAILABILITY STATEMENT}

The raw data supporting the conclusions of this article will be made available by the authors, without undue reservation.

\section{REFERENCES}

Abe, K., Miyoshi, K., Muto, T., Ruspita, I., Horiguchi, T., Nagata, T., et al. (2007). Establishment and characterization of rat dental epithelial derived ameloblast-lineage clones. J Biosci Bioeng 103, 479-485. doi: 10.1263/jbb.10 3.479

Aldred, M. J., Savarirayan, R., and Crawford, P. J. (2003). Amelogenesis imperfecta: a classification and catalogue for the 21st century. Oral Dis 9, 19-23. doi: 10.1034/j.1601-0825.2003.00843.x

\section{ETHICS STATEMENT}

The animal study was reviewed and approved by The Forsyth Institute and Massachusetts General Hospital Boston, Boston MA, United States.

\section{AUTHOR CONTRIBUTIONS}

IR and MB contributed to the concept and design of the study. IR, PD, YX, and $\mathrm{MB}$ contributed to the acquisition of data. IR, PD, SK, and MB prepared the figures. IR, PD, RD'S, and $\mathrm{MB}$ contributed to the data analysis and interpretation. IR, PD, and MB wrote the manuscript. IR, PD, MS, TN, RD'S, and MB contributed to the editing and the critical revision for intellectual content. All authors have approved the final version of the submitted manuscript.

\section{FUNDING}

This study was supported by funds from NIH [grants R01DE027255-01S1 (subcontract), R21DE028091], to MB and (grant R01DE027255), to RD'S. The funders had no role in study design, data collection and analysis, decision to publish, or preparation of the manuscript.

\section{SUPPLEMENTARY MATERIAL}

The Supplementary Material for this article can be found online at: https://www.frontiersin.org/articles/10.3389/fphys. 2020.582610/full\#supplementary-material

Supplementary Figure $1 \mid$ Msx2 directly binds to Msx2 recognition sites on the Sp6 promoter: In silico model showing six potential Msx2 binding sites (F1-R1: -1317 to -1323; F2-R2: -1731 to -1736; F3-R3: -1881 to 1886; F4-R4: -2109 to $-2114,-2119$ to $-2124,-2141$ to -2146 ; F5-R5: -2487 to -2493 ; F6-R6, position -2827 to -2833 ) on the Sp6 promoter using MatInspector. The different binding sites are represented by green ellipses. Primers were designed from different promoter regions (blue arrows). After chromatin immunoprecipitation, samples from LS8 cells transfected with pCMV-Msx2-FLAG were PCR amplified; the binding region was directly amplified prior to immunoprecipitation (1\% Input) and specifically amplified in the immunoprecipitated sample (anti-FLAG). (right panel). No amplification was detected in the normal mouse serum IgG-immuno precipitated sample (IgG; negative control). Pol A is the positive control. Band in the input lane shows endogenous binding while band in the sample lane shows binding after specific immunoprecipitation with FLAG tagged antibody after overexpression. The results show that Msx2 binds only to four out of six predicted regions in the Sp6 promoter.

Babajko, S., De La Dure-Molla, M., Jedeon, K., and Berdal, A. (2014). MSX2 in ameloblast cell fate and activity. Front Physiol 5:510. doi: 10.3389/fphys.2014. 00510

Bartlett, J. D. (2013). Dental enamel development: proteinases and their enamel matrix substrates. ISRN Dent 2013:684607.

Bei, M. (2009a). Molecular genetics of ameloblast cell lineage. J Exp Zool B Mol Dev Evol 312B, 437-444. doi: 10.1002/jez.b.21261

Bei, M. (2009b). Molecular genetics of tooth development. Curr. Opin. Genet. Dev 19, 504-510. doi: 10.1016/j.gde.2009.09.002 
Bei, M., and Maas, R. (1998). FGFS and BMP4 induce both Msxl-independent and Msxl-dependent signaling pathways in early tooth development. Development 125, 4325-4333.

Bei, M., Stowell, S., and Maas, R. (2004). Msx2 controls ameloblast terminal differentiation. Dev. Dyn. 231, 758-765. doi: 10.1002/dvdy.20182

Bouwman, P., Gollner, H., Elasser, H. P., Eckhoff, G., Karis, A., Grosveld, F., et al. (2000). Transcription factor Sp3 is essential for post-natal survival and late tooth development. EMBO J. 19, 655-661. doi: 10.1093/emboj/19.4.655

Catron, K. M., Iler, N., and Abate, C. (1993). Nucleotides flanking a conserved TAAT core dictate the DNA binding specificity of three murine homeodomain proteins. Mol. Cell. Biol. 13, 2354-2365. doi: 10.1128/mcb.13.4.2354

Catron, K. M., Zhang, H., Marshall, S. C., Inostroza, J. A., Wilson, J. M., and Abate, C. (1995). Transcriptional repression by Msx-1 does not require homeodomain DNA-binding sites. Mol. Cell. Biol. 15, 861-871. doi: 10.1128/mcb.15.2.861

Chang, H., Wang, Y., Liu, H., Nan, X., Wong, S., Peng, S., et al. (2017). Mutant Runx2 regulates amelogenesis and osteogenesis through a miR-185-5p-Dlx2 axis. Cell Death Dis 8:3221.

Chen, L. S., Couwenhoven, R. I., Hsu, D., Luo, W., and Snead, M. L. (1992). Maintenance of amelogenin gene expression by transformed epithelial cells of mouse enamel organ. Arch. Oral. Biol. 37, 771-778. doi: 10.1016/0003-9969(92) 90110-t

Diamond, E., Amen, M., Hu, Q., Espinoza, H. M., and Amendt, B. A. (2006). Functional interactions between Dlx2 and lymphoid enhancer factor regulate Msx2. Nucleic Acids Res. 34, 5951-5965. doi: 10.1093/nar/gkl689

Duval, N., Daubas, P., Bourcier de Carbon, C., St Cloment, C., Tinevez, J. Y., Lopes, M., et al. (2014). Msx1 and Msx2 act as essential activators of Atoh1 expression in the murine spinal cord. Development 141, 1726-1736. doi: 10. 1242/dev.099002

Gritli-Linde, A., Bei, M., Maas, R., Zhang, X. M., Linde, A., and Mcmahon, A. P. (2002). Shh signaling within the dental epithelium is necessary for cell proliferation, growth and polarization. Development 129, 5323-5337. doi: 10 . 1242/dev.00100

Habelitz, S. (2015). Materials engineering by ameloblasts. J. Dent. Res. 94, 759-767. doi: 10.1177/0022034515577963

Hu, J. C., Hu, Y., Smith, C. E., Mckee, M. D., Wright, J. T., Yamakoshi, Y., et al. (2008). Enamel defects and ameloblast-specific expression in Enam knockout/lacz knock-in mice. J. Biol. Chem. 283, 10858-10871.

Huang, Z., Sargeant, T. D., Hulvat, J. F., Mata, A., Bringas, P. Jr., Koh, C. Y., et al. (2008). Bioactive nanofibers instruct cells to proliferate and differentiate during enamel regeneration. J. Bone. Miner. Res. 23, 1995-2006. doi: 10.1359/jbmr. 080705

Kollar, E. J., and Lumsden, A. G. (1979). Tooth morphogenesis: the role of the innervation during induction and pattern formation. J Biol Buccale 7, 49-60.

MacKenzie, A., Ferguson, M. W., and Sharpe, P. T. (1992). Expression patterns of the homeobox gene, Hox-8, in the mouse embryo suggest a role in specifying tooth initiation and shape. Development 115, 403-420.

Molla, M., Descroix, V., Aioub, M., Simon, S., Castaneda, B., Hotton, D., et al. (2010). Enamel protein regulation and dental and periodontal physiopathology in MSX2 mutant mice. Am. J. Pathol. 177, 2516-2526. doi: 10.2353/ajpath.2010. 091224

Nakamura, T., De Vega, S., Fukumoto, S., Jimenez, L., Unda, F., and Yamada, Y. (2008). Transcription factor epiprofin is essential for tooth morphogenesis by regulating epithelial cell fate and tooth number. J. Biol. Chem. 283, 4825-4833. doi: $10.1074 /$ jbc.m708388200

Nanci, A. (2007). Development, Structure, and Function. Ten Cate's Oral Histology. St.Louis, MO: Mosby Inc.

Ruspita, I., Miyoshi, K., Muto, T., Abe, K., Horiguchi, T., and Noma, T. (2008). Sp6 downregulation of follistatin gene expression in ameloblasts. J. Med. Invest. 55, 87-98. doi: 10.2152/jmi.55.87
Satokata, I., Ma, L., Ohshima, H., Bei, M., Woo, I., Nishizawa, K., et al. (2000). Msx2 deficiency in mice causes pleiotropic defects in bone growth and ectodermal organ formation. Nat. Genet. 24, 391-395. doi: 10.1038/74231

Shin, N. Y., Yamazaki, H., Beniash, E., Yang, X., Margolis, S. S., Pugach, M. K., et al. (2020). Amelogenin phosphorylation regulates tooth enamel formation by stabilizing a transient amorphous mineral precursor. J. Biol. Chem. 295, 1943-1959. doi: 10.1074/jbc.ra119.010506

Smith, C. E. L., Whitehouse, L. L. E., Poulter, J. A., Wilkinson Hewitt, L., Nadat, F., Jackson, B. R., et al. (2020). A missense variant in specificity protein 6 (SP6) is associated with amelogenesis imperfecta. Hum. Mol. Genet. 29, 1417-1425. doi: $10.1093 / \mathrm{hmg} / \mathrm{ddaa} 041$

Suda, N., Kitahara, Y., and Ohyama, K. (2006). A case of amelogenesis imperfecta, cleft lip and palate and polycystic kidney disease. Orthod. Craniofac. Res. 9, 52-56. doi: 10.1111/j.1601-6343.2006.00337.x

Thesleff, I. A., and Nieminen, P. (2005). Tooth Induction. Chichester: John Wiley \& Sons Ltd.

Utami, T. W., Miyoshi, K., Hagita, H., Yanuaryska, R. D., Horiguchi, T., and Noma, T. (2011). Possible linkage of SP6 transcriptional activity with amelogenesis by protein stabilization. J Biomed Biotechnol 2011:320987.

Wang, X. P., Suomalainen, M., Jorgez, C. J., Matzuk, M. M., Werner, S., and Thesleff, I. (2004). Follistatin regulates enamel patterning in mouse incisors by asymmetrically inhibiting BMP signaling and ameloblast differentiation. Dev. Cell 7, 719-730. doi: 10.1016/j.devcel.2004.09.012

Wright, J. T. (2006). The molecular etiologies and associated phenotypes of amelogenesis imperfecta. Am J Med Genet A 140, 2547-2555. doi: 10.1002/ ajmg.a.31358

Wright, J. T., Carrion, I. A., and Morris, C. (2015). The molecular basis of hereditary enamel. J Dent Res. 94, 52-61. doi: 10.1177/0022034514556708

Wright, J. T., Hart, P. S., Aldred, M. J., Seow, K., Crawford, P. J., Hong, S. P., et al. (2000). Identification of CCAAT/enhancer-binding protein alpha as a transactivator of the mouse amelogenin gene. J. Biol. Chem. 275, 12273-12280. doi: $10.1074 /$ jbc. 275.16 .12273

Xu, L., Harada, H., Yokohama-Tamaki, T., Matsumoto, S., Tanaka, J., and Taniguchi, A. (2006). Reuptake of extracellular amelogenin by dental epithelial cells results in increased levels of amelogenin MRNA through enhanced MRNA stabilization. J. Biol. Chem. 281, 2257-2262. doi: 10.1074/jbc.m507695200

Zhang, H., Hu, G., Wang, H., Sciavolino, P., Iler, N., Shen, M. M., et al. (1997). Heterodimerization of Msx and Dlx homeoproteins results in functional antagonism. Mol. Cell. Biol. 17, 2920-2932. doi: 10.1128/mcb.17.5. 2920

Zhao, M., Gupta, V., Raj, L., Roussel, M., and Bei, M. (2013). A network of transcription factors operates during early tooth morphogenesis. Mol. Cell Biol. 33, 3099-3112. doi: 10.1128/MCB.00524-13

Zhou, Y. L., Lei, Y., and Snead, M. L. (2000). Functional antagonism between Msx2 and CCAAT/enhancer-binding protein alpha in regulating the mouse amelogenin gene expression is mediated by protein-protein interaction. J. Biol. Chem. 275, 29066-29075.

Conflict of Interest: The authors declare that the research was conducted in the absence of any commercial or financial relationships that could be construed as a potential conflict of interest.

Copyright (c) 2020 Ruspita, Das, Xia, Kelangi, Miyoshi, Noma, Snead, D'Souza and Bei. This is an open-access article distributed under the terms of the Creative Commons Attribution License (CC BY). The use, distribution or reproduction in other forums is permitted, provided the original author(s) and the copyright owner(s) are credited and that the original publication in this journal is cited, in accordance with accepted academic practice. No use, distribution or reproduction is permitted which does not comply with these terms. 